\title{
Continuous hemodiafiltration as a rescue therapy for patients with cardiopulmonary failure caused by enterovirus-71: a retrospective observational study in a PICU
}

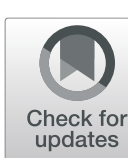

Chunxia Wang ${ }^{1,2+}$, Yun Cui ${ }^{1 \dagger}$, Yan Zhu ${ }^{1}$, Fei Wang ${ }^{1}$, Qunfang Rong ${ }^{1}$ and Yucai Zhang ${ }^{1,2^{*}}$ [D

\begin{abstract}
Background: Hand, foot and mouth disease (HFMD) remains a burdensome health issue in mainland China. Enterovirus71 (EV-A71) is the main pathogen of severe HFMD. Continuous hemofiltration improves fluid overload, restores kidney function and alleviates inflammatory reactions. The aim of the present study was to evaluate the effects of continuous veno-venous hemodiafiltration (CWHDF) on severe HFMD caused by EV-A71(EV-A71-HFMD) in a pediatric intensive care unit (PICU).
\end{abstract}

Methods: A retrospective observational study was performed in a tertiary university PICU from January 2012 to December 2016. Children with severe EV-A71-HFMD complicated by cardiopulmonary failure were included. The patients were divided into a CWHDF group and a conventional therapy (control) group (non-CWHDF). The demographics, characteristics, and outcomes between the groups were collected and analyzed.

Results: Twenty-nine patients with severe EV-A71-HFMD were enrolled. The 28-day mortality was 17.6\% (3/17) in the CWHDF group and 33.3\% (4/12) in the non-CWHDF group, with no statistical significance between the two groups $(P=0.403)$. The median interval between CWHDF initiation and PICU admission was $6(4,8.5) \mathrm{hrs}$, and the median duration of CWHDF was $48(36,64)$ hrs. The left ventricular ejection fraction (LVEF) and cardiac index $(C I)$ in the CWHDF group were improved after treatment. The plasma levels of catecholamines and renin-angiotensinaldosterone system (RAAS) substances in the CWHDF group were significantly decreased after treatment. The decreased catecholamines and RAAS substances included adrenalin $(169.8$ [145.5, 244.6] vs. 148.0 [109.0, 208.1] ng/L, $P=0.033)$, dopamine (152.7 [97.0, 191.1] vs. $96.0[68.0,160.9] \mathrm{ng} / \mathrm{L}, P=0.026)$, angiotensin II $(185.9[125.2,800.0]$ vs. $106.0[90.8,232.5] \mathrm{ng} / \mathrm{L}, P=0.047)$, aldosterone (165.7 [94.0, 353.3] vs. $103.3[84.3,144.3] \mathrm{ng} / \mathrm{L}, P=0.033)$, and renin $(1.12[0.74,3.45]$ vs. $0.79[0.52,1.25] \mu \mathrm{g} / \mathrm{L} / \mathrm{h}, P=0.029)$,

Conclusions: CWHDF reduced the levels of catecholamines and RAAS substances and improved cardiovascular function. Continuous hemodiafiltration may represent a potential therapy in patients with severe EV-A71-HFMD complicated with cardiopulmonary failure.

Keywords: Enterovirus71, Hand, foot and mouth disease, Cardiopulmonary failure, Continuous veno-venous hemodiafiltration, Mortality, Child

\footnotetext{
* Correspondence: zyucai2018@163.com

${ }^{\dagger}$ Chunxia Wang and Yun Cui contributed equally to this work.

'Department of Critical Care Medicine, Shanghai Children's Hospital,

Shanghai Jiao Tong University, No.355 Luding Road, Shanghai 200062, Putuo

District, China

${ }^{2}$ Institute of Pediatric Critical Care, Shanghai Jiao Tong University, Shanghai

200062, China
}

(c) The Author(s). 2019 Open Access This article is distributed under the terms of the Creative Commons Attribution 4.0 International License (http://creativecommons.org/licenses/by/4.0/), which permits unrestricted use, distribution, and reproduction in any medium, provided you give appropriate credit to the original author(s) and the source, provide a link to the Creative Commons license, and indicate if changes were made. The Creative Commons Public Domain Dedication waiver (http://creativecommons.org/publicdomain/zero/1.0/) applies to the data made available in this article, unless otherwise stated. 


\section{Background}

Hand, foot and mouth disease (HFMD) has become a major global health issue, and HFMD in China accounted for $87 \%$ (9.8 million/11.3 million) of all HFMD cases reported to the World Health Organization (WHO) from 2010 to 2014 [1]. Cardiopulmonary failure is the main cause of mortality in children with HFMD under the age of 3 years [2]. In 2012, a large outbreak of HFMD occurred over a wide area in China, and enterovirus71 (EVA71) was responsible for $60.4 \%$ of HFMD inpatients and $88.5 \%$ of severe cases with cardiopulmonary failure [3]. According to the China Guidelines for the diagnosis and treatment of severe HFMD due to EV-A71 [4], severe HFMD with cardiopulmonary failure caused by EV-A71, referred to as stage 3-4 EV-A71-HFMD, remains a lifethreatening and challenging disease in a pediatric intensive care units (PICUs) in China.

Continuous renal replacement therapy (CRRT) is available for pediatric critically ill patients. Moreover, CRRT is suitable for renal replacement, fluid overload (FO) management, homeostasis stabilization, toxin removal, and proinflammatory cytokine release in infants and young children $[5,6]$. Children with severe HFMD develop rapidly progressing sympathetic hyperactivity, pulmonary edema, and cardiopulmonary failure [7, 8]. Inappropriate fluid resuscitation and acute kidney injury (AKI) may lead to FO in cases of severe HFMD. Limited studies have observed that hemofiltration is be an efficient rescue treatment in children with severe HFMD [9-11].CRRT has been empirically used in patients with severe EV-A71-HFMD with cardiopulmonary failure since 2012 in our PICU. Our previous studies indicated that continuous hemofiltration effectively reduced the inflammatory response and improved organ dysfunction in patients with severe sepsis $[12,13]$ or secondary hemophagocytic lymphohistiocytosis [14]. Thus, we suspected that hemofiltration could be a potential therapy for severe EV-A71HFMD complicated by cardiopulmonary failure.

Tachycardia and systemic hypertension are symptoms of ulminant EV-A71-related HFMD and are considered to be a sympathetic storm [15]. An excited sympathetic tone with a catecholamine surge might contribute to extremely high systemic vascular resistance, cardiac dysfunction, and passive pulmonaryedema [16]. In the present study, we retrospectively analyzed the medical records of patients with severe EV-A71-HFMD complicated by cardiopulmonary failure. The benefits of continuous veno-venous hemodiafiltration (CVVHDF) on mortality and clinical parameters were analyzed. We speculated that catecholamines and renin-angiotensin-aldosterone system (RAAS) substances may play key roles in the process of cardiopulmonary failure caused by EV-A71 in patients with stage 3 or 4 HFMD. Therefore, we measured the levels of epinephrine, dopamine, rennin, angiotensin II and aldosterone in patients with stages 3 or 4 HFMD.

\section{Methods}

Study design

A retrospective observational study was performed in patients with severe EV-A71-HFMD complicated with cardiopulmonary failure admitted to the PICU at Shanghai Children's Hospital between January 2012 and December 2016. According to whether the patients received CVVHDF during PICU hospitalization, the patients were divided into a non-CVVHDF group, who underwent conventional therapy, or a CVVHDF group, who underwent conventional therapy plus CVVHDF. The study was conducted in accordance with the ethical principles of the Declaration of Helsinki (and subsequent revisions) and the current standards for observational studies. This study was approved by the Ethics Review Committee, Children's Hospital of Shanghai/Shanghai Children's Hospital, Shanghai Jiao Tong University and conducted in accordance with the provisions of the Declaration of Helsinki (Approval No. 2016R010-F01). The need for informed consent to participate was waived because we used deidentified retrospective data.

\section{Patients}

Diagnosis and staging were performed according to the clinical therapy expert consensus on severe cases caused by EV-A71. Patients with severe EV-A71-HFMD complicated with cardiopulmonary failure were diagnosed as having stage 3 or 4 [4]. The symptoms of patients in stage 3 or 4 include increased heart and respiratory rates, cold sweats, cold extremities, mottled skin, increased blood pressure, tachycardia (bradycardia wasalso occasionally seen), tachypnea, cyanosis, cough with pink foamy or bloody sputum, hypotension and ultimately cardiovascular collapse [4].

The inclusion criteria were as follows: (1) patients aged 1 month to 14 years old; (2) patients with severe stage 3 or 4 EV-A71-HFDM, defined by the clinical therapy expert consensus on severe cases caused by EV-A71 (4); and (3) patients who were EV-A71 IgM or RT-PCR positive. Patients who required cardiopulmonary resuscitation and died within $24 \mathrm{~h}$ after admission were excluded.

The conventional management for HFDM was performed according to the 2008 Guidelines for the diagnosis and the expert consensus on the rescue and treatment of severe cases caused by EV-A71 [4, 17]. The physiological fluid requirement was $60-80 \mathrm{ml} / \mathrm{kg} /$ day in the absence of deliberate diuresis. Patients with shock were resuscitated with normal saline $10-20 \mathrm{ml} / \mathrm{kg} /$ time over $30 \mathrm{~min}$ while administering vasoactive agents $[4,17]$. The hemodynamic change in stage 3 was characterized by high dynamicity and high resistance. Milrinone was used with a loading dosage of $50-75 \mu \mathrm{g} / \mathrm{kg}$. The maintenance dose was $0.25-$ $0.75 \mu \mathrm{g} / \mathrm{kg} / \mathrm{min}$. The total duration of the infusion perioddid not exceed $72 \mathrm{~h}$. Phentolamine $(1-20 \mu \mathrm{g} / \mathrm{kg} / \mathrm{min})$ or 
sodium nitroprusside $(0.5-5 \mu \mathrm{g} / \mathrm{kg} / \mathrm{min})$ was initiated at a low dose and gradually increased to an appropriate dose level to control blood pressure to a level below that of constituting severe hypertension at the corresponding age if necessary. When hypotension manifested in patients with stage 4 EV-A71-HFDM, positive inotropic agents and vasopressors, such as dopamine at $5-20 \mu \mathrm{g} / \mathrm{kg} / \mathrm{min}$, norepinephrine at $0.05-2 \mu \mathrm{g} / \mathrm{kg} / \mathrm{min}$, adrenalineat $0.05-$ $2 \mu \mathrm{g} / \mathrm{kg} /$ minute and dobutamine at $2.5-20 \mu \mathrm{g} / \mathrm{kg} / \mathrm{min}$ were used. If patients had suffered from nosocomial infection or were supported by mechanical ventilation for more than 3 days, antibiotics were used.

\section{Continuous hemofiltration}

The indications for CVVHDF initiation in our study included an $\mathrm{FO}>10 \%[\mathrm{FO}=(\mathrm{CVVHDF}$ initial weight-PICU admission weight)/PICU admission weight $\times 100 \%$ ], acute kidney injury (AKI), or unstable hemodynamics, such as cardiogenic shock shock and multiple organ dysfunction $[18,19]$. For patients with severe EV-A71-HFMD presenting with refractory cardiovascular disorder, CVVHDF as an empirical adjuvant therapy combined with conventional treatment was administered to maintain stable hemodynamics. Patients who suffered from severe coagulopathy disorder (international normalized ratio [INR] $>3.0$ or platelet count $<10 \times 10^{9} / \mathrm{L} ; n=6$ ) or a history of biofilm or hemofilter allergy $(n=1)$ or patients without parental consent for $\operatorname{CRRT}(n=5)$ were treated with conventional therapy and included in the non-CVVHDF group.

CVVHDF was performed ata flow rate of $35-50 \mathrm{~mL} / \mathrm{kg} /$ hr. for both ultrafiltrate and dialysate using a PRISMA or PRISMA flex blood purification machine and Gambro PRISMA M60 membrane hemofilter equipped with an AN69 (Gambro Renal Products, Meyzieu, France). Vascular access was obtained with an $8 \mathrm{~F}$ central venous catheter (GamCath; Gambro, Colombes, France) in the right internal jugular or femoral vein according to the patient's body weight. Blood flow was set at a constant $4-6 \mathrm{~mL} / \mathrm{kg} /$ min to achieve a filtration fraction of $25-35 \%$. The replacement fluid was prepared according to the modified Ports formula and contained $\mathrm{Na}^{+} 130 \mathrm{mmol} / \mathrm{L}, \mathrm{K}^{+} 4$ $\mathrm{mmol} / \mathrm{L}, \mathrm{HCO}_{3}{ }^{-} 28 \mathrm{mmol} / \mathrm{L}, \mathrm{Ca}^{2+} 1.5 \mathrm{mmol} / \mathrm{L}, \mathrm{Mg}^{2+} 3.2$ $\mathrm{mmol} / \mathrm{L}, \mathrm{Cl}^{-} 109 \mathrm{mmol} / \mathrm{L}$, and glucose $0.2 \mathrm{~g} / \mathrm{L}$. The preto postdilution ratio was 1:2. The rate of replacement fluid was $35 \mathrm{ml} / \mathrm{kg} / \mathrm{h} \mathrm{(Qf)} \mathrm{or} 10-15 \mathrm{ml} / \mathrm{min}$ (Qd). The filter circuit was pretreated with saline that contained 5000-10, $000 \mathrm{IU} / \mathrm{L}$ unfractionated heparin. During CVVHDF, unfractionated heparin was used with at aninfusion rate of $5-20 \mathrm{U} / \mathrm{kg} / \mathrm{h}$. The activated partial thromboplastin time (APTT) was detected every $4-6 \mathrm{~h}$, and the transmembrane pressure was modified between 50 and $120 \mathrm{mmHg}$. The dose of heparin was regulated to maintain APTT at 1.52 -fold the normal value. The hemofilter was changed every $24 \mathrm{~h}$ or when clotted. The patients with severe coagulation disorders (APTT $>80$ s or INR $>3.0$ ), a biofilm allergic reaction, or difficult venin catheter access were managed with conventional therapies, as in the control group. The implementation of CVVHDF for cardiovascular indications was fully explained, and the parents of the patients provided written informed consent prior to CVVHDF initiation.

In the present study, the indications for the weaning of CVVHDF included the following: (1) a normal heart rate and blood pressure; (2) serum lactate $<2 \mathrm{mmol} / \mathrm{L}$; and (3) a urine output more than $1 \mathrm{ml} / \mathrm{kg} / \mathrm{h}$ and $\mathrm{FO}<$ $10 \%$. The primary endpoints were as follows: (1) ameliorated hyperthermia; (2) improved pulmonary edema and pulmonary effusion; (3) a urine output of $>1 \mathrm{ml} / \mathrm{kg} / \mathrm{h}$; and (4) a normal blood pressure. Continuous hemofiltration was terminated when the following conditions occurred: (1) the children developed severe bleeding or uncontrollable hemorrhaging; or (2) symptoms were not obviously improved after $72 \mathrm{~h}$. The assessments for initiation and weaning, as well as the effects of CVVHDF, were performed by an attending intensivist in our PICU.

\section{Data collection and definitions}

All data were retrieved from the hospital database system. Variables were defined prior to data collection and were entered in a standardized format during data collection. The collected data included demographic data (such as age, sex, and body weight), details of the initial presentation on admission, clinical features for EV-A71-induced HFMD, length of PICU stay, vital signs (temperature, heart rate, and blood pressure), cardiac parameters (left ventricular ejection fraction, LVEF; and cardiac index, CI), the ratio of the partial pressure of oxygen in arterial blood $\left(\mathrm{PaO}_{2}\right)$ to the inspired oxygen fraction $\left(\mathrm{FiO}_{2}\right)\left(\mathrm{PaO}_{2} / \mathrm{FiO}_{2}\right)$ before and after CVVHDF, as well as 28-day mortality, duration of mechanical ventilation, and duration of vasoactive agent administration. Cardiac ultrasound was performed by a specialized cardiologist. Laboratory data, including $\mathrm{pH}$, creatinine ( $\mathrm{Cr}$ ), alanine transaminase (ALT), serum lactate (Lac), cardiac troponin I (cTnI) and creatine kinase-MB (CK-MB), as well as catecholamine-like substances, including adrenalin, dopamine, renin, angiotensin II, and aldosterone, were obtained from the computerized hospital medical database. Biochemical indexes were collected at admission and after 3 days in the non-CVVHDF group. Biochemical indexes were collected before CVVHDF initiation and after CVVHDF for 3 days in the CVVHDF group.

\section{Statistical analysis}

The data were analyzed using SPSS (v. 22.0) (SPSS Inc., Chicago, IL). All the variables were tested for normal distribution using the Kolmogorov-Smirnov test. Continuous variables with abnormal distributions were summarized as 
medians (IQRs). The Mann-Whitney $U$ test was used to compare the continuous variables with abnormal distributions. The chi-square test was used to compare the categorical data. A value of $P<0.05$ was considered statistically significant.

\section{Results}

Baseline characteristics of patients with SevereEV-A71HFMD with cardiopulmonary failure

Twenty-nine patients with severe EV-A71-HFMD complicated with cardiopulmonary failure were included. All patients received mechanical ventilation (29/29). Twelve patients were managed with conventional therapy, and 17 patients were treated with conventional therapy plus CVVHDF. There was no significant difference in age, sex, body weight, temperature, heart rate, blood pressure, LVEF, CI or the use of vasoactive agents between the non-CVVHDF group and the CVVHDF group. There was no significant difference in the incidence of $\mathrm{AKI}$ and FO between the non-CVVHDF group and the CVVHDF group ( 2 cases vs. 4 cases, $P=0.653 ; 3$ cases vs. 6 cases, $P=0.694$; respectively) (Table 1 ).
Outcomes and laboratory indexes of patients in the nonCVVHDF group and the CVVHDF group

The levels of $\mathrm{pH}(P=0.842), \mathrm{PaO}_{2} / \mathrm{FiO}_{2}(P=0.757)$, lactate $(P=0.249), \mathrm{Cr}(P=0.757)$, ALT $(P=0.894)$, c'TnI $(P=0.877)$ and $\mathrm{CK}-\mathrm{MB}(P=0.308)$ were not significantly different between the two groups. The median interval between CVVHDF initiation and PICU admission was 6 $(4,8.5)$ hrs. The duration of vasoactive agent administration and the length of stay in the PICU were significantly decreased in the CVVHDF group compared with the non-CVVHDF group $(P=0.024, P=0.030$, respectively; Table 2). Moreover, the total 28-day mortality of severe EV-A71-HFMD was $4 / 12$ in the non-CVVHDF group and $3 / 17$ in the CVVHDF group, without a significant difference $(P=0.403$, Table 2$)$. The duration of mechanical ventilation had a decreased tendency in the CVVHD group compared with the non-CVVHDF group, but the difference was not significant $(P=0.063$, Table 2$)$.

\section{Effects of CVVHDF}

The median duration of CVVHDF was $48(36,64)$ hrs. Fever $(P=0.001)$, heart rate $(P=0.001)$, systolic blood pressure $(P=0.003), \operatorname{LVEF}(P=0.017)$ and $C I(P=0.002)$

Table 1 Baseline characteristics of patients with severe HFMD caused by EV71 in the non-CWHDF group and the CWHDF group

\begin{tabular}{|c|c|c|c|}
\hline Characteristics & $\begin{array}{l}\text { Non-CWHDF group } \\
(n=12)\end{array}$ & $\begin{array}{l}\text { CWHDF group } \\
(n=17)\end{array}$ & $P$ \\
\hline Age, month & $21.0(8.3,23.8)$ & $23.0(12.5,24.0)$ & 0.372 \\
\hline Male, $n$ & 8 & 10 & 0.717 \\
\hline Severity & & & 0.979 \\
\hline Stage $3, \mathrm{n}$ & 7 & 10 & \\
\hline Stage $4, \mathrm{n}$ & 5 & 7 & \\
\hline Body weight, kg & $10.6(8.3,14.4)$ & $11.0(10.0,14.4)$ & 0.565 \\
\hline Body temperature, ${ }^{\circ} \mathrm{C}$ & $39.7(39.4,39.8)$ & $39.7(39.3,40.0)$ & 0.518 \\
\hline Heart rate, bpm & $188(171,195)$ & $187(178,198)$ & 0.982 \\
\hline LVEF, \% & $38(26,44)$ & $37(24,47)$ & 0.773 \\
\hline $\mathrm{Cl}, \mathrm{L} / \mathrm{min} / \mathrm{m}^{2}$ & $2.89(2.23,3.63)$ & $2.73(1.92,3.95)$ & 0.642 \\
\hline Systolic blood pressure, $\mathrm{mmHg}$ & $132.0(76.0,150.5)$ & $139(102,155)$ & 0.116 \\
\hline Acute kidney injury, $\mathrm{n}$ & 2 & 4 & 0.653 \\
\hline Fluid overload, n & 3 & 6 & 0.694 \\
\hline Mechanical ventilator, $\mathrm{n}$ & 12 & 17 & / \\
\hline \multicolumn{4}{|l|}{ Vasoactive agents } \\
\hline Milrinone, $\mathrm{n}$ & 7 & 7 & 0.462 \\
\hline Phentolamine, $\mathrm{n}$ & 1 & 0 & 0.414 \\
\hline Sodium nitroprusside, $\mathrm{n}$ & 4 & 2 & 0.198 \\
\hline Dopamine, $\mathrm{n}$ & 4 & 6 & 0.913 \\
\hline Dobutamine, $\mathrm{n}$ & 9 & 13 & 1.000 \\
\hline Norepinephrine, n & 1 & 3 & 0.622 \\
\hline Adrenaline, $\mathrm{n}$ & 2 & 4 & 1.000 \\
\hline
\end{tabular}

LVEF Left ventricular ejection fraction, Cl Cardiac index. Continuous variables were summarized as the median (IQR) 
Table 2 Outcomes and laboratory indexes in patients with severe HFMD caused by EV71 in the non-CWHDF group and the CWHDF group

\begin{tabular}{|c|c|c|c|}
\hline Characteristics & $\begin{array}{l}\text { Non-CWHDF group } \\
(\mathrm{n}=12)\end{array}$ & $\begin{array}{l}\text { CWHDF group } \\
(n=17)\end{array}$ & $P$ \\
\hline \multicolumn{4}{|l|}{ Biochemical data } \\
\hline $\mathrm{PH}$ & $7.21(6.94,7.37)$ & $7.23(7.07,7.31)$ & 0.842 \\
\hline $\mathrm{PaO}_{2} / \mathrm{FiO}_{2}, \mathrm{mmHg}$ & $134(112.5,157.8)$ & $135(101.5,160)$ & 0.757 \\
\hline $\mathrm{Lac}, \mathrm{mmol} / \mathrm{L}$ & $4.35(3.20,5.80)$ & $3.5(2.8,3.9)$ & 0.249 \\
\hline $\mathrm{Cr}, \mu \mathrm{mol} / \mathrm{L}$ & $58.0(28.5,79.8)$ & $48(28,77)$ & 0.757 \\
\hline$A L T, I U / L$ & $36.0(19.8,63.3)$ & $35(13,70)$ & 0.894 \\
\hline$c T n l, \mu g / L$ & $0.16(0.06,0.38)$ & $0.12(0.06,0.39)$ & 0.877 \\
\hline CK-MB, IU/L & $35(21,73)$ & $37(26,112)$ & 0.308 \\
\hline \multicolumn{4}{|l|}{ Outcomes } \\
\hline Duration of mechanical ventilation, day & $14.5(11.5,20.3)$ & $8.0(5.5,17.5)$ & 0.063 \\
\hline Duration of vasoactive agents, day & $7.5(6.0,10.0)$ & $5.0(4.5,7.0)$ & 0.024 \\
\hline Stay in PICU, day & $23(17,27)$ & $12(9,22)$ & 0.030 \\
\hline 28-day death, $n$ & 4 & 3 & 0.403 \\
\hline
\end{tabular}

$\mathrm{PaO}_{2} / \mathrm{FiO}_{2}$ The ratio of the partial pressure of oxygen in arterial blood $\left(\mathrm{PaO}_{2}\right)$ to the inspired oxygen fraction $\left(\mathrm{FiO}_{2}\right)$, Lac Lactate, $\mathrm{Cr}$ Creatinine, ALT Alanine transaminase, cTnI Cardiac troponin I, CK-MB Creatine kinase-MB. Continuous variables were summarized as the median (IQR)

were significantly improved after treatment in the CVVHDF group (Table 3). The levels of blood lactate were significantly decreased in the CVVHDF group $(3.50$ $[2.75,3.90]$ vs. $1.3[0.95,1.90] \mathrm{mmol} / \mathrm{L}, P=0.001$, Table 3$)$. Moreover, the levels of adrenalin (169.8 [145.5, 244.6] vs. 148.0 [109.0, 208.1] ng/L, $P=0.033)$, dopamine (152.7 [97.0, 191.1] vs. $96.0[68.0,160.9] \mathrm{ng} / \mathrm{L}, P=0.026)$, renin (1.12 [0.74, 3.45] vs. $0.79[0.52,1.25] \mu \mathrm{g} / \mathrm{L} \cdot \mathrm{h}, P=0.029)$, angiotensin II (185.9 [125.2, 800.0] vs. 106.0 [90.8, 232.5] ng/L, $P=0.047)$, and aldosterone (165.7 [94.0, 353.3] vs. $103.3[84.3,144.3] \mathrm{ng} / \mathrm{L}, P=0.033)$ were significantly decreased after CVVHDF (Table 3, Fig. 1). In the nonCVVHDF group, fever $(P=0.001)$ and heart rate $(P=$ 0.001 ) were significantly improved, and the levels of blood lactate were significantly decreased after $72 \mathrm{~h}$ of conventional treatment $(4.35[3.20,5.80]$ vs. $2.2[1.93,3.75]$ $\mathrm{mmol} / \mathrm{L}, P=0.014$, Table 3$)$. Moreover, the $\mathrm{pH}$ level improved (7.21 [6.94, 7.37] vs. 7.31 [7.21, 7.38], $P=0.015$ ). However, the levels of adrenalin, dopamine, renin, angiotensinII and aldosterone did not change after 3 days of treatment in the non-CVVHDF group $(P=0.190, P=$ $0.678, P=0.872, P=0.215$, and $P=0.730$, respectively, Table 3).

\section{Six-month follow-up assessments}

During a 6-month follow-up, 1 patient in the CVVHDF group died due to central respiratory failure. One patient in the CVVHDF group was discharged with difficulty swallowing but recovered. The other patients were alive without comorbidities at the time of follow-up.

\section{Discussion}

Patients with severe EV-A71-HFMD complicated with cardiopulmonary failure have a high risk of mortality. In the present study, we demonstrated the benefits of CVVHDF in improving cardiopulmonary function and decreasing the levels of catecholamines and RAAS substances, which were associated with a decreased tendency of 28-day mortality in severe EV-A71-HFMD patients. To our knowledge, this study is the first report regarding the impact of CVVHDF on EV-A71-HFMD.

A previous report indicated that the mortality associated with EV-A71-induced severe HFMD in the PICU was $30.4 \%$ (7/23) during the 2010 ongoing outbreak in the Shanghai region [3]. A recent study showed that the mortality rate of severe HFMD was $21.3 \%$ (19/89) in the PICU at the Children's Hospital of Chongqing Medical University of China from June 2015 to September 2016 [20], suggesting an improvement in the management of severe HFMD in China in recent years. In the present study, the 28-day mortality rate of severe EV-A71HFMD was lower in the CVVHDF group than in the non-CVVHDF group (3/17 vs. 4/12, $P=0.403)$, implying that the application of CVVHDF might contribute to the decreased tendency in mortality. In our previous study, CVVHDF showed clinical benefits in the management of severe sepsis, sepsis-associated hemophagocytic lymphohistiocytosis, and sepsis-associated liver injury in our PICU [12-14, 21]. The current study focused on CVVHDF empiric therapy for severe EV-A71-HFMD, as CVVHDF has been a recommend therapy for severe pediatric EV-A71HFMD complicated by cardiopulmonary failure in the 2018 Guideline for HFMD in China [4]. This warrants further 
Table 3 Changes of characteristics of severe HFMD caused by EV71 in the non-CWHDF group and the CWHDF group

\begin{tabular}{|c|c|c|c|c|c|c|c|}
\hline \multirow[t]{2}{*}{ Variables } & \multicolumn{3}{|l|}{$\begin{array}{l}\text { Non-CWHDF group } \\
(n=12)\end{array}$} & \multicolumn{3}{|l|}{$\begin{array}{l}\text { CWHDF group } \\
(n=17)\end{array}$} & \multirow[t]{2}{*}{$P^{\prime \prime}$} \\
\hline & 1st day (A1) & 3rd day (A2) & P & Before CWHDF (B1) & $72 \mathrm{~h}$ after CWHDF (B2) & $P^{\prime}$ & \\
\hline \multicolumn{8}{|l|}{ Vital signs } \\
\hline Heart rate, bpm & $188.0(170.8,194.8)$ & $142.0(130.3,157.0)$ & 0.001 & $187.0(178.0,197.5)$ & $134.0(122.5,147.0)$ & 0.001 & 0.143 \\
\hline Body temperature, ${ }^{\circ} \mathrm{C}$ & $39.7(39.4,39.8)$ & $38.0(37.3,38.5)$ & 0.001 & $39.7(39.3,40.0)$ & $36.8(36.4,37.3)$ & 0.001 & 0.001 \\
\hline Systolic blood pressure, mmHg & $132.0(76.0,150.5)$ & $121.5(103.5,140.3)$ & 0.854 & $139(101.5,155.0)$ & $102.5(86.5,115.7)$ & 0.003 & 0.001 \\
\hline \multicolumn{8}{|l|}{ Cardiac parameters } \\
\hline LVEF, \% & $38(26,44)$ & $41(29,46)$ & 0.204 & $37(21,47)$ & $55(21,62)$ & 0.017 & 0.184 \\
\hline $\mathrm{Cl}, \mathrm{L} / \mathrm{min} / \mathrm{m}^{2}$ & $2.89(2.23,3.63)$ & $3.11(2.22,3.73)$ & 0.621 & $2.73(1.92,3.95)$ & $4.77(1.92,5.75)$ & 0.002 & 0.199 \\
\hline$c T n l, \mu g / L$ & $0.16(0.06,0.38)$ & $0.08(0.04,0.15)$ & 0.032 & $0.12(0.06,0.39)$ & $0.02(0.01,0.48)$ & 0.365 & 0.325 \\
\hline CK-MB, IU/L & $35.0(20.5,72.8)$ & $33.0(19.0,64.3)$ & 0.240 & $37.0(25.5,112.0)$ & $78.0(39.0,110.5)$ & 0.298 & 0.008 \\
\hline \multicolumn{8}{|l|}{ Organ function indexes } \\
\hline Blood glucose, mg/dL & $9.85(6.98,12.18)$ & $7.9(6.95,10.35)$ & 0.311 & $9.4(7.9,11.3)$ & $6.1(5.7,11.8)$ & 0.118 & 0.057 \\
\hline $\mathrm{Lac}, \mathrm{mmol} / \mathrm{L}$ & $4.35(3.20,5.80)$ & $2.2(1.93,3.75)$ & 0.014 & $3.50(2.75,3.90)$ & $1.3(0.95,1.90)$ & 0.001 & 0.005 \\
\hline $\mathrm{pH}$ & $7.21(6.94,7.37)$ & $7.31(7.21,7.38)$ & 0.015 & $7.23(7.07,7.31)$ & $7.39(7.37,7.44)$ & 0.001 & 0.041 \\
\hline $\mathrm{Cr}, \mu \mathrm{mol} / \mathrm{L}$ & $58.0(28.5,79.8)$ & $61.0(54.5,119.5)$ & 0.121 & $48.0(28.0,77.0)$ & $38.0(20.5,72.0)$ & 0.517 & 0.023 \\
\hline $\mathrm{ALT}, \mathrm{IU} / \mathrm{L}$ & $36.0(19.8,63.3)$ & $63.0(27.0,140.8)$ & 0.048 & $35.0(12.5,70.0)$ & $38.0(26.0,52.0)$ & 0.227 & 0.170 \\
\hline \multicolumn{8}{|l|}{ Catecholamine } \\
\hline Adrenalin, ng/L & $153.6(127.0,184.4)$ & $145.4(85.8,175.1)$ & 0.190 & $169.8(145.5,244.6)$ & $148.0(109.0,208.1)$ & 0.033 & 0.223 \\
\hline Dopamine, ng/L & $127(82.4,156.5)$ & $133.4(83.0,171.7)$ & 0.678 & $152.7(97.0,191.1)$ & $96.0(68.0,160.9)$ & 0.026 & 0.319 \\
\hline \multicolumn{8}{|l|}{ RAAS substances } \\
\hline Renin, $\mu \mathrm{g} / \mathrm{L} . \mathrm{h}$ & $2.35(1.49,4.76)$ & $2.90(1.30,4.80)$ & 0.872 & $1.12(0.74,3.45)$ & $0.79(0.52,1.25)$ & 0.029 & 0.003 \\
\hline Angiotensinll, ng/L & $348.4(131.5,773.0)$ & $565.4(210.9,773.3)$ & 0.215 & $185.9(125.2,800.0)$ & $106.0(90.8,232.5)$ & 0.047 & 0.001 \\
\hline Aldosterone, ng/L & $211.2(119.8,362.6)$ & $281.9(167.9,349.0)$ & 0.730 & $165.7(94.0,353.3)$ & $103.3(84.3,144.3)$ & 0.033 & 0.001 \\
\hline
\end{tabular}

LVEF Left ventricular ejection fraction, Cl Cardiac index, CTnI Cardiac troponin I, CK-MB Creatine kinase-MB, Lac Lactate, Cr Creatinine, ALT Alanine transaminase. Continuous variables were summarized as the median (IQR). $P$ indicates $A 1$ vs. $A 2 ; P^{\prime}$ indicates $B 1$ vs. $B 2 ; P^{\prime \prime}$ indicates $A 2$ vs. $B 2$

research using well-designed prospective trials with a large population.

Our previous study on severe EV-A71-HFMD indicated that the involved organ systems affected by EV-A71 included the central nervous system, the respiratory system, and the cardiovascular system [22]. In the present study, 29 patients with EV-A71-HFMD displayed high heart rates and high lactate levels on PICU admission, and all patients received mechanical ventilation for respiratory failure or cardiogenic pulmonary edema. Limited studies found that EV-A71-infected patients with autonomic nervous system dysregulation and/or pulmonary edema had high levels of norepinephrine (NE) and epinephrine (EP) [23], and plasma NE gradually increased with the aggravation of the disease [24]. In the present study, we observed that the blood levels of catecholamines, including adrenalin and dopamine, decreased after treatment in the CVVHDF group but not in the non-CVVHDF group. To date, the effects of continuous hemofiltration on the removal of catecholamines are controversial. Bellomo and colleagues [25] found no significant amounts of catecholamines in the ultrafiltrate of critically ill patients with AKI who received CRRT. Inconsistently, the ultrafiltrate from patients with heart failureproduced a positive inotropic response potentially attributable to catecholamines [26]. Furthermore, Siebeck $M$ et al. [27] indicated that the catecholamine concentration was significantly decreased after continuous hemofiltration. Given that adrenalin (molecular weight: 219) and dopamine (molecular weight: 153) are "small" molecules, we speculated that adrenalin and dopamine may be removed directly by CVVHDF. Because EVA71-induced catecholamine storms play a crucial role in cardiopulmonary failure [28], the reduction of catecholamines by CVVHDF might contribute to the improvement of cardiopulmonary failure in patients with severe EV-A71-HFMD.

To date, there is little information available regarding RAAS involvement in EV-A71-HFMD. Whether CVVHDF removed RAAS substances resulting in an improvement in cardiopulmonary failure requires further investigation. To our knowledge, we are the first to report that the levels of renin, angiotensin II, and aldosterone were significantly decreased in patients in the CVVHDF group. Several studies indicated that the RAAS blockade reduced cardiovascular 


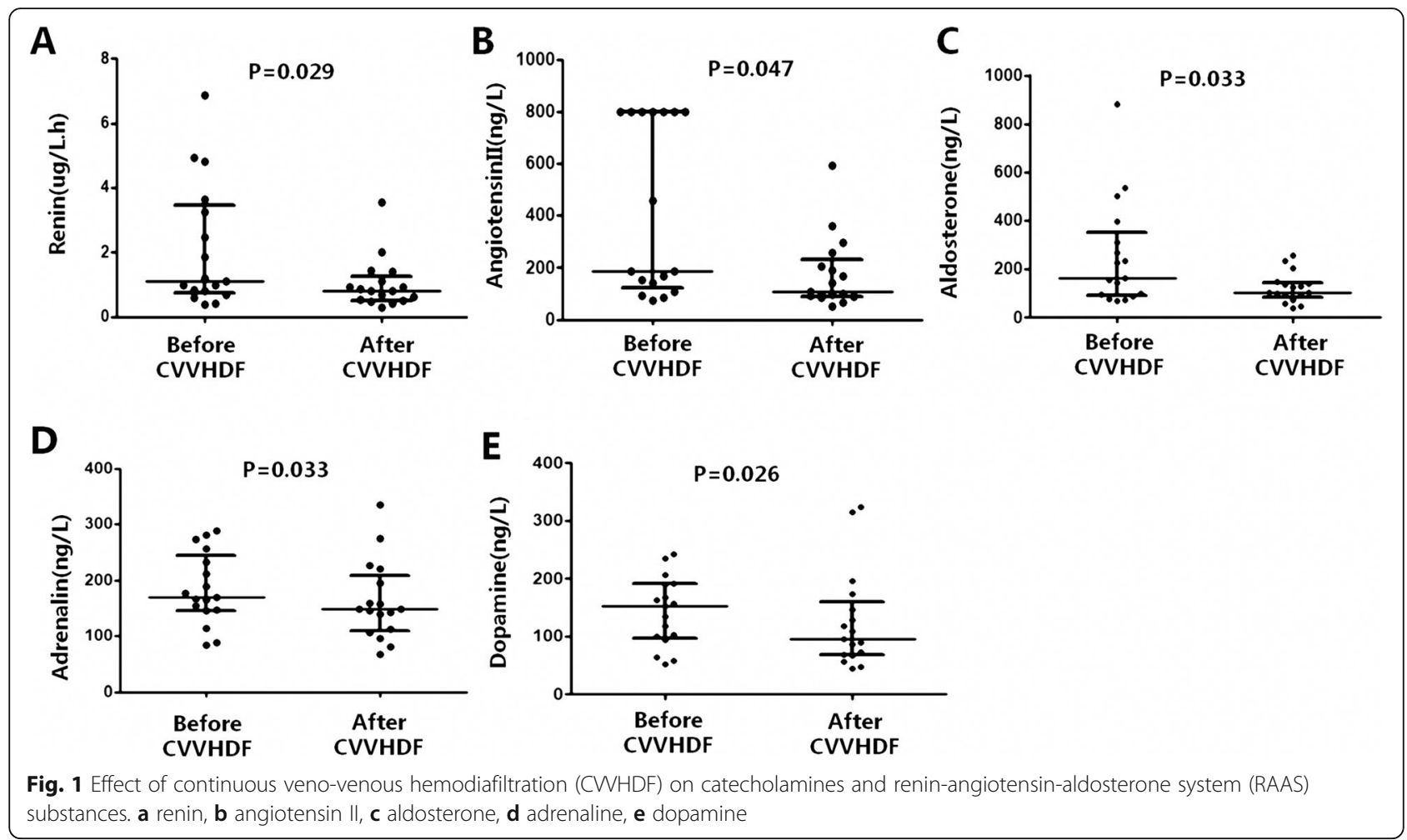

morbidity and mortality, and angiotensin converting enzyme inhibitor therapy was used in patients with left ventricular dysfunction $[29,30]$. Therefore, we speculated that RAAS components might contribute to the protective effects of CVVHDF on cardiopulmonary failure in severe EVA71-HFMD patients.

Our study has several limitations. First, only 17 patients received CVVHDF in our retrospective study, which affected the power of the conclusion. Second, an analysis of the filtered solution is necessary to identify the role of CVVHDF in removing catecholamines and RAAS substances. Third, the difference in the CK-MB levels after treatment between the nonCVVHDF and CVVHDF groups needs further investigation in a large population. Nevertheless, our results are noteworthy because CVVHDF showed a tendency to improve 28-day mortality in severe EV-A71-HFMD patients. This result warrants further research using well-designed prospective trials based on a large population.

\section{Conclusions}

CVVHDF represents a potential therapy in patients with severe EV-A71-HFMD complicated with cardiopulmonary failure; CVVHDF improved cardiovascular function associated with reduced levels of adrenaline, dopamine, as well as RAAS substances including rennin, angiotensin II, and aldosterone.

\section{Abbreviations}

AKI: Acute kidney injury; ALT: Alanine transaminase; APTT: Activated partial thromboplastin time; Cl: Cardiac index; CK-MB: Creatine kinase-MB;

Cr: Creatinine; CRRT: Continuous renal replacement therapy; CTnl: Cardiac troponin I; CWHDF: Continuous veno-venous hemodiafiltration; EV-

A71: Enterovirus71; EV-A71-HFMD: Severe HFMD caused by EV-A71; FO: Fluid overload; HFMD: Hand, foot and mouth disease; INR: International normalized ratio; Lac: Lactate; LVEF: Left ventricular ejection fraction; $\mathrm{PaO}_{2} /$ $\mathrm{FiO}_{2}$ : The ratio of the partial pressure of oxygen in arterial blood $\left(\mathrm{PaO}_{2}\right)$ to the inspired oxygen fraction $\left(\mathrm{FiO}_{2}\right)$; PICU: Pediatric intensive care unit

\section{Acknowledgements}

None.

Authors' contributions

CW and YZhang conceived and designed the study. YZhu, YC, and FW collected and analyzed the data. QR, YC and YZhu contributed analysis tools and discussion. CW, YC and YZhang wrote the paper. All authors have read and approved the manuscript

\section{Funding}

This study was supported by the New Advanced Technology Project at the Shanghai City Hospital Development Center (SHDC12014116), the Multicenter Clinical Research Program of Shanghai Jiao Tong University School of Medicine(DLY201618), Science and Technology Commission of Shanghai Municipality (17411968900, 16411970300), and Shanghai Municipal Education Commission-Gaofeng Clinical Medicine grant support (20171928).Chunxia Wang was supported by the Talents Program of Shanghai Jiao Tong University School of Medicine (17XJ11018). The funders had no role in the study design, data collection or analysis, decision to publish, or preparation of the manuscript.

Availability of data and materials

Our present study was a retrospective observational study. All the data were obtained from the medical records of patients. All the data generated or analyzed during this study are available from the corresponding author upon reasonable request. 


\section{Ethics approval and consent to participate}

This study was approved byEthics Review Committee, Children's Hospital of Shanghai/Shanghai Children's Hospital, Shanghai Jiao Tong University and conducted in accordance with the provisions of the Declaration of Helsinki (ApprovalNo.2016R010-F01).The need for informed consent to participate was waived because we used deidentified retrospective data. All the data were obtained from the medical records of patients. All data generated or analyzed during this study are available from the corresponding author upon reasonable request.

\section{Consent for publication}

Not applicable.

\section{Competing interests}

The authors declare that they have no competing interests exist.

Received: 25 December 2018 Accepted: 27 September 2019

Published online: 21 October 2019

\section{References}

1. Wu JT, Jit M, Zheng $Y$, et al. Routine pediatric enterovirus 71 vaccination in China: a cost-effectiveness analysis. PLoS Med. 2016;13:e1001975.

2. Yang SD, Li PQ, Li YM, et al. Clinical manifestations of severe enterovirus 71 infection and early assessment in a southern China population. BMC Infect Dis. 2017;17:153

3. Zhang Q, MacDonald NE, Smith JC, et al. Severe enterovirus type 71 nervous system infections in children in the Shanghai region of China: clinical manifestations and implications for prevention and care. Pediatr Infect Dis J. 2014:33:482-7.

4. Li XW, Ni X, Qian SY, et al. Chinese guidelines for the diagnosis and treatment of hand, foot and mouth disease (2018 edition). World J Pediatr. 2018;14:437-47.

5. Goldstein SL. Continuous renal replacement therapy: mechanism of clearance, fluid removal, indications and outcomes. CurrOpinPediatr. 2011;23:181-5.

6. Honore PM, Jacobs R, Joannes-Boyau O, et al. Newly designed CRRT membranes for sepsis and SIRS--a pragmatic approach for bedside intensivists summarizing the more recent advances: a systematic structured review. ASAIO J. 2013;59:99-106.

7. Wu JM, Wang JN, Tsai YC, et al. Cardiopulmonary manifestations of fulminant enterovirus 71 infection. Pediatrics. 2002;109:E26.

8. McMinn P, Stratov I, Nagarajan L, et al. Neurological manifestations of enterovirus 71 infection in children during an outbreak of hand, foot, and mouth disease in Western Australia. Clin Infect Dis. 2001;32:236-42.

9. Cao L, Geng W, Xu M, et al. Effect of continuous hemofiltration on inflammatory mediators and hemodynamics in children with severe hand, foot and mouth disease. Zhongguo Dang Dai Er Ke Za Zhi. 2016;18:219-23 Article in Chinese.

10. Nguyen NT, Pham HV. Hoang CQ, et al. Epidemiological and clinical characteristics of children who died from hand, foot and mouth disease in Vietnam, 2011. BMC Infect Dis. 2014;14:341.

11. Qui P, Khanh T, Trieu H, et al. Intravenous magnesium sulfate for the management of severe hand, foot, and mouth disease with autonomic nervous system dysregulation in Vietnamese children: study protocol for a randomized controlled trial. Trials. 2016;17:98.

12. Miao $\mathrm{H}$, Wang $F$, Xiong $\mathrm{X}$, et al. Clinical benefits of high-volume hemofiltration in critically ill pediatric patients with severe sepsis: aretrospective cohort study. Blood Purif. 2018:45:18-27.

13. Miao H, Shi J, Wang $\mathrm{C}$, et al. Continuous renal replacement therapy in pediatric severe sepsis: a propensity score-matched prospective multicenter cohort study in the PICU. Crit Care Med. 2019. https://doi.org/10.1097/CCM. 0000000000003901

14. Cui Y, Zhang YC, Kang YL, et al. High-volume hemofiltration in critically ill patients with secondary hemophagocytic lymphohistiocytosis/macrophage activation syndrome: a prospective study in the PICU. PediatrCrit Care Med. 2016;17:e437-43

15. Chang LY, Lin TY, Hsu KH, et al. Clinical features and risk factors of pulmonary oedema after enterovirus-71-related hand, foot, and mouth disease. Lancet. 1999:354:1682-6.

16. Huang $F$, Jan $S$, Chen $P$, et al. Left ventricular dysfunction in children with fulminant enterovirus 71 infection: an evaluation of the clinical course. Clin Infect Dis. 2002;34:1020-4.
17. Clinical Experts Group of the Ministry of Health for Hand, Foot, and Mouth Disease. Experts consensus on rescue and treatment of severe cases with enterovirus 71 (EV71) infection. ZhonghuaErKe Za Zhi. 2011;49:675-8 Article in Chinese.

18. Anzai T, Lai NC, Gao M, et al. Dissociation between regional dysfunction and beta-adrenergic receptor signaling in heart failure. Am J Physiol. 1998; 275:H1267-73.

19. Zhao $P$, Zheng $R$, Xue L, et al. Early fluid resuscitation and high volumehemofiltration decrease septic shock progression in swine. Biomed Res Int 2015:2015:181845.

20. Dang HX, Liu CJ, Li J, et al. Clinical significance and prognostic effect of serum 25-hydroxyvitamin D concentrations in critical and severe hand, foot and mouth disease. Nutrients. 2017;9:pii: E478.

21. Cui Y, Xiong $X$, Wang F, et al. Continuous hemofiltration improves the prognosis of bacterial sepsis complicated by liver dysfunction in children. BMC Pediatr. 2018:18:269.

22. Zhang Y, Li X, Zhu X, et al. Clinical characteristics and treatment of severe encephalitis associated with neurogenic pulmonary edema caused by enterovirus 71 in China. World J Emerg Med. 2010;1:108-13.

23. Liao YT, Wang SM, Wang JR, et al. Norepinephrine andepinephrineenhanced the infectivity of enterovirus 71. PLoS One. 2015; 10:e0135154.

24. Lei Z, Li B, Li H, et al. Relationship between catecholamine level and gene polymorphism of $\beta 1$ adrenergic receptor G1165C in children with EV71 infection in hand foot and mouth disease. Asian Pac J Trop Med. 2017;10:473-7.

25. Bellomo R, McGrath B, Boyce N. Effect of continuous venovenous hemofiltration with dialysis on hormone and catecholamine clearance in critically ill patients with acute renal failure. Crit Care Med. 1994;22:833-7.

26. Blake P, Hasegawa Y, Khosla MC, et al. Isolation of "myocardial depressant factor(s)" from the ultrafiltrate of heart failure patients with acute renal failure. ASAIO J. 1996;42:M911-5.

27. Siebeck M, Dimski T, Brandenburger T, et al. Super high-flux continuous venovenoushemodialysisusing regional citrate anticoagulation: long-term stability of middle molecule clearance. TherApher Dial. 2018:22:355-64.

28. Victorio $C B, X u Y, N g$, et al. A clinically authentic mouse model of enterovirus 71(EV-A71) -induced neurogenic pulmonary oedema. Sci Rep. 2016;6:28876.

29. Morena M, Delbosc S, Dupuy AM, et al. Overproduction of reactive oxygen species in end-stage renal disease patients: a potential component of hemodialysis-associated inflammation. Hemodial Int. 2005;9:37-46.

30. Patten RD, Kronenberg MW, Benedict CR, et al. Acute and long-term effects of the angiotensin-converting enzyme inhibitor, enalapril, on adrenergic activity and sensitivity during exercise in patients with left ventricular systolic dysfunction. Am Heart J. 1997;134:37-43.

\section{Publisher's Note}

Springer Nature remains neutral with regard to jurisdictional claims in published maps and institutional affiliations.

Ready to submit your research? Choose BMC and benefit from:

- fast, convenient online submission

- thorough peer review by experienced researchers in your field

- rapid publication on acceptance

- support for research data, including large and complex data types

- gold Open Access which fosters wider collaboration and increased citations

- maximum visibility for your research: over $100 \mathrm{M}$ website views per year

At BMC, research is always in progress.

Learn more biomedcentral.com/submissions 